\title{
Therapeutic window of CPR duration for target temperature management in OHCA survivors
}

Objectives: We investigated the cardiopulmonary resuscitation (CPR) duration effective for target temperature management (TTM) in out-of-hospital cardiac arrest (OHCA) survivor.

Methods: We conducted a retrospective analysis of prospective multicenter registered data of $1045 \mathrm{OHCA}$ patients who had sustained return of spontaneous circulation (ROSC) in three academic emergency centers from June 2014 to August 2019.

- No-flow time (NFT) and low-flow time (LFT) were defined as the time from cardiac arrest to the start of CPR and the time from the start of CPR to ROSC, respectively.

- The post-resuscitation care was divided into two groups; a group that maintained the core temperature from 32 to $36^{\circ} \mathrm{C}$ with temperature feedback system (TTM group) and a group that maintained normal body temperature with fever control (Non-TTM group).

- Primary outcome is good cerebral performance category (CPC 1 or 2) scale at 1 months.

Results: 500 OHCA survivors were excluded from the analysis, A total of $545 \mathrm{OHCA}$ survivors were finally included in the analysis. OHCA survivors with good CPC who had CPR duration from 6 and 10 min with NFT and from 0 to 10 min with LFT were statistically significantly higher in the TTM group than Non-TTM group.

\section{Conclusion:}

The effective therapeutic window for the TTM might be from 6 to 10 minutes with NFT and 0 to 10 minutes with LFT in post-resuscitation care of OHCA survivor. Further prospective study using the randomized control trial or large scale retrospective studies using the propensity score matching will be needed.
Figure 1. Flow chart of enrolled patients according to the diagnostic methods

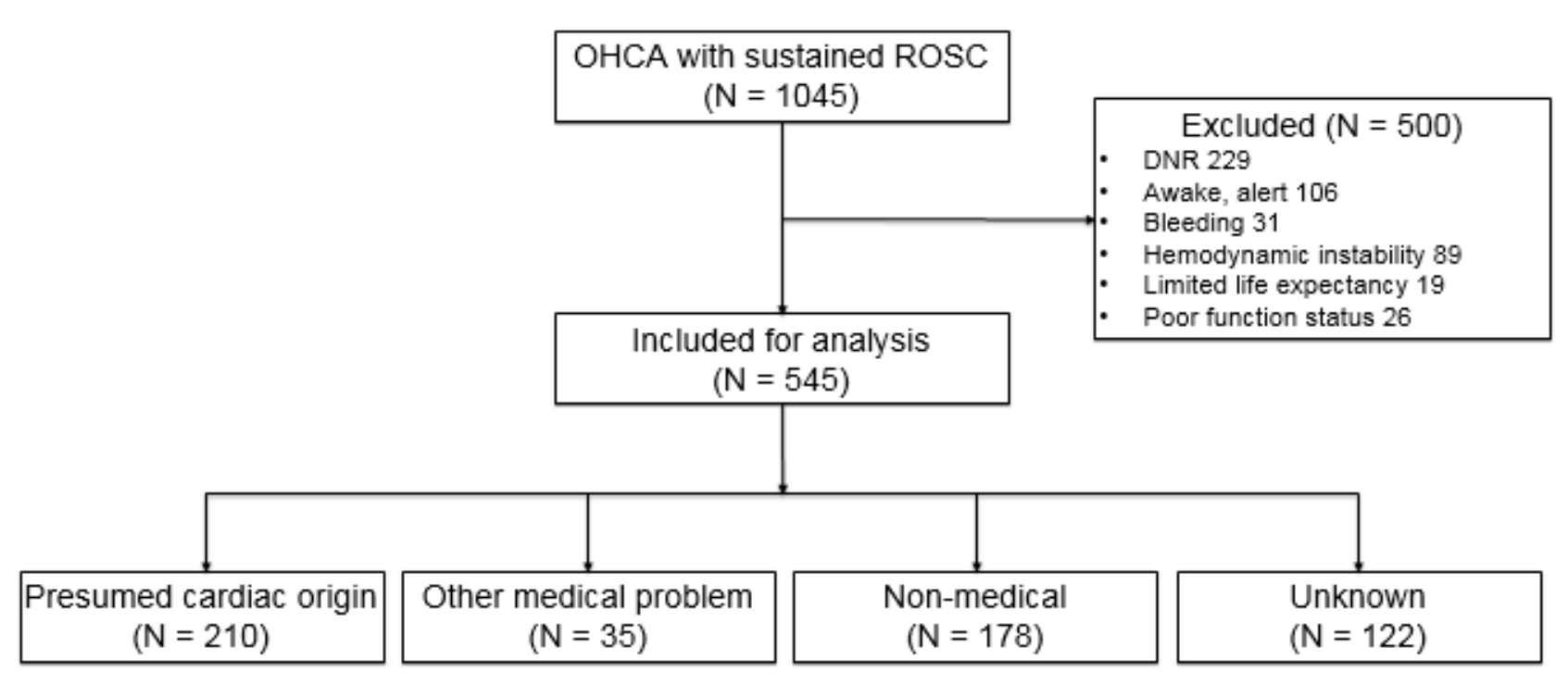

\begin{tabular}{|c|c|c|c|c|c|c|c|c|}
\hline \multicolumn{9}{|c|}{ Table 1. CPC by NFT in 5 minutes division } \\
\hline $\begin{array}{c}\text { Durati } \\
\text { on }\end{array}$ & $\begin{array}{c}\text { Total } \\
\mathrm{n}=\end{array}$ & TTM & $\mathrm{n}=$ & Good & $\%$ & $\mathrm{Bad}$ & $\%$ & $\begin{array}{c}\mathrm{P}- \\
\text { value }\end{array}$ \\
\hline \multirow{2}{*}{$0-5$} & \multirow{2}{*}{361} & Yes & 227 & 59 & 26 & 168 & 74 & \multirow{2}{*}{0.208} \\
\hline & & No & 134 & 27 & 20.1 & 107 & 79.9 & \\
\hline \multirow{2}{*}{$6-10$} & \multirow{2}{*}{105} & Yes & 81 & 12 & 14.8 & 69 & 85.2 & \multirow{2}{*}{0.045} \\
\hline & & No & 24 & 0 & 0 & 24 & 100 & \\
\hline \multirow{2}{*}{$11-15$} & \multirow{2}{*}{36} & Yes & 24 & 3 & 12.5 & 21 & 87.5 & \multirow{2}{*}{0.733} \\
\hline & & No & 12 & 2 & 16.7 & 10 & 83.3 & \\
\hline \multirow{2}{*}{$16-20$} & \multirow{2}{*}{22} & Yes & 16 & 1 & 6.2 & 15 & 93.8 & \multirow{2}{*}{0.449} \\
\hline & & No & 6 & 1 & 16.7 & 5 & 83.3 & \\
\hline \multirow{2}{*}{$21-$} & \multirow{2}{*}{12} & Yes & 8 & 0 & 0 & 8 & 100 & \multirow{2}{*}{1} \\
\hline & & No & 4 & 0 & 0 & 4 & 100 & \\
\hline
\end{tabular}

\begin{tabular}{|c|c|c|c|c|c|c|c|c|}
\hline \multicolumn{9}{|c|}{ Table 2. CPC by LFT in 10 minutes division } \\
\hline $\begin{array}{c}\text { Durati } \\
\text { on }\end{array}$ & $\begin{array}{c}\text { Total } \\
\mathrm{n}=\end{array}$ & TTM & $\mathrm{n}=$ & Good & $\%$ & Bad & $\%$ & $\begin{array}{c}\mathrm{P}- \\
\text { value }\end{array}$ \\
\hline \multirow{2}{*}{$0-10$} & \multirow{2}{*}{94} & Yes & 64 & 32 & 50 & 32 & 50 & \multirow{2}{*}{0.033} \\
\hline & & No & 30 & 8 & 26.7 & 22 & 73.3 & \\
\hline \multirow{2}{*}{$11-20$} & \multirow{2}{*}{119} & Yes & 80 & 23 & 23 & 28.8 & 57 & \multirow{2}{*}{0.951} \\
\hline & & No & 39 & 11 & 11 & 28.2 & 28 & \\
\hline \multirow{2}{*}{$21-30$} & \multirow{2}{*}{141} & Yes & 95 & 13 & 13 & 13.7 & 82 & \multirow{2}{*}{0.807} \\
\hline & & No & 46 & 7 & 7 & 15.2 & 39 & \\
\hline \multirow{2}{*}{$31-40$} & \multirow{2}{*}{101} & Yes & 71 & 3 & 3 & 4.2 & 68 & \multirow{2}{*}{0.834} \\
\hline & & No & 30 & 1 & 1 & 3.3 & 29 & \\
\hline \multirow{2}{*}{$41-50$} & \multirow{2}{*}{32} & Yes & 20 & 1 & 1 & 5 & 19 & \multirow{2}{*}{0.706} \\
\hline & & No & 12 & 1 & 1 & 8.3 & 11 & \\
\hline \multirow{2}{*}{$51-60$} & \multirow{2}{*}{21} & Yes & 12 & 1 & 1 & 8.3 & 11 & \multirow{2}{*}{0.375} \\
\hline & & No & 9 & 0 & 0 & 0 & 9 & \\
\hline \multirow{2}{*}{$61-$} & \multirow{2}{*}{23} & Yes & 15 & 2 & 2 & 13.3 & 13 & \multirow{2}{*}{0.28} \\
\hline & & No & 8 & 0 & 0 & 0 & 8 & \\
\hline
\end{tabular}

Rate of Good CPC according to the NFT

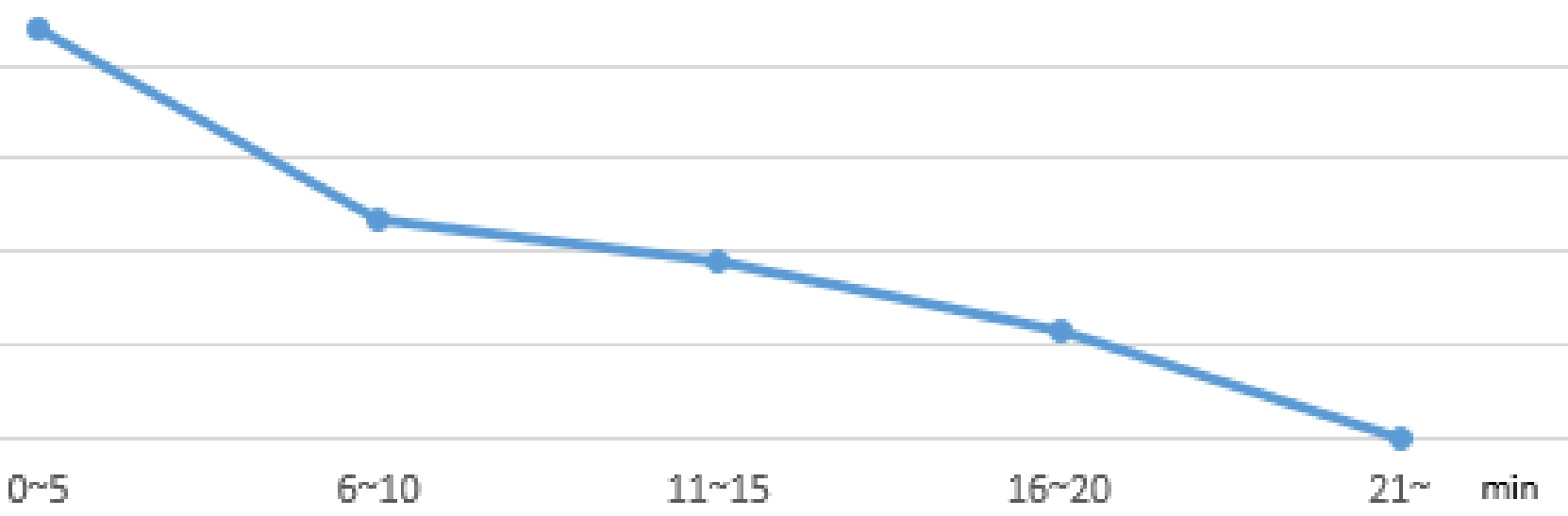

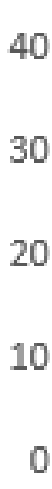

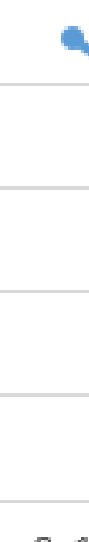

$0 \sim 10$

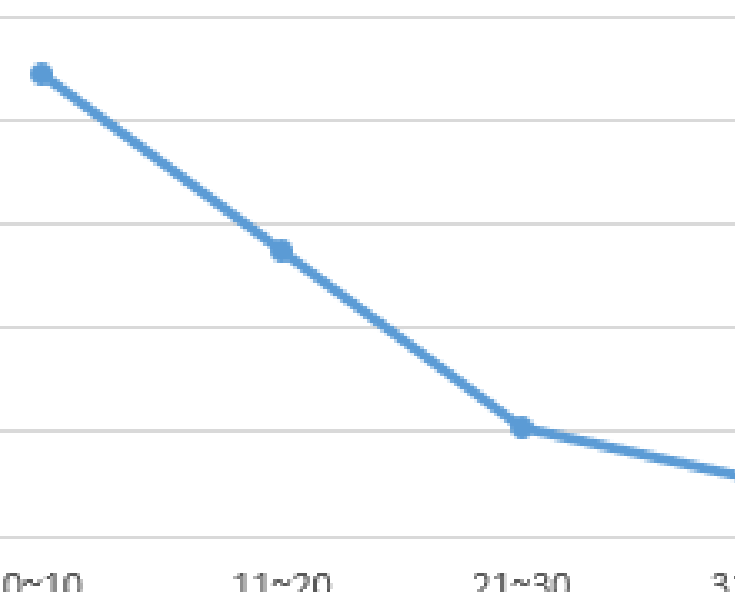

$11 \sim 20$ 